\title{
PARAMETRIC DESIGN OF INJECTORS FOR LDI-3 COMBUSTORS
}

\author{
Kumud Ajmani \\ Vantage Partners, LLC \\ Cleveland, Ohio, USA
}

\author{
Hukam Mongia \\ Purdue University \\ West Lafayette, Indiana, USA
}

\author{
Phil Lee \\ Woodward FST, Inc \\ Zeeland, MI, USA
}

\subsection{ABSTRACT}

Application of a partially calibrated National Combustion Code (NCC) for providing guidance in the design of the $3^{\text {rd }}$ generation of the Lean-Direct Injection (LDI) multi-element combustion configuration (LDI-3) is summarized. NCC was used to perform non-reacting and two-phase reacting flow computations on several LDI-3 injector configurations in a single-element and a five-element injector array. All computations were performed with a consistent approach for mesh-generation, turbulence, spray simulations, ignition and chemical kinetics-modeling. Both qualitative and quantitative assessment of the computed flowfield characteristics of the several design options led to selection of an optimal injector LDI3 design that met all the requirements including effective area, aerodynamics and fuel-air mixing criteria. Computed LDI-3 emissions (namely, NOx, CO and UHC) will be compared with the prior generation LDI2 combustor experimental data at relevant engine cycle conditions.

\subsection{INTRODUCTION}

NASA's Environmentally Responsible Aviation (ERA) program has revived interest in defining the $2^{\text {nd }}$ generation LDI concepts (LDI-2) for meeting the $\mathrm{N}+2$ technology goals of emissions and performance. The emissions and performance characteristics for NASA Glenn led LDI efforts for the $1^{\text {st }}$ generation (LDI1) are summarized by [Tacina 2005] followed by the $2^{\text {nd }}$ generation LDI-2 by [Tacina 2014] and [Zink 2014].

Experimental measurements of emissions for axial-swirler LDI-2 configurations involving both pressure atomizing and airblast injectors of 13-element sector designs were performed at Woodward FST (for low $\mathrm{P}_{3}$ ) and at NASA Glenn Research Center (GRC) for moderately high $\mathrm{P}_{3}$ conditions [Tacina 2014]. The empirical design of the three LDI-2 injectors was based on the experience of the nine-element LDI-1 arrays involving axial-swirlers [Tacina 2005]. The experimental activities on LDI-1 and LDI-2 along with empirical correlation development effort have recently been complemented by NCC calibration works with both single and multi-element configurations, as summarized by [Ajmani 2013] for LDI-1, and [Ajmani 2014a, 2014b] for two LDI-2 configurations.

NCC is a reacting flow CFD computational code formulated, developed and validated at NASA Glenn Research Center (GRC). NCC has been used extensively to analyze a single-injector LDI geometry [Liu 2011, Liu 2013] and multiple-injector LDI-1 configurations [Davoudzadeh 2006, Iannetti 2008, Ajmani 2013]. These works helped establish best practices for CFD analysis of LDI systems with NCC, similar to FLUENT-based methodology summarized by [Mongia 2008] for application of CFD in combustion system design and development process. The best practices evolved through LDI-1 computations provided the basis for calibrating NCC with the LDI-2 data as summarized by [Ajmani 2014a], a first publication that is planned to be followed by some future articles describing more extensive calibration with all the three LDI2 configurations tested at NASA.

This paper provides a summary on the use of the partially calibrated NCC for performing parametric evaluation of several candidate LDI-3 configurations. The primary goal of the work was to use CFD to help optimize the LDI-3 injector design toward defining a final design hardware that will be machined and delivered for flame-tube testing at both NASA GRC and Woodward FST, Inc. NCC has been used to compute heat release, NOx and CO emissions for optimized LDI-3 design(s) proposed by Woodward FST, Inc. (WFST). The predicted CFD predictions for the recommended LDI-3 design will be compared with the planned experimental database. 


\subsection{COMPUTATIONAL APPROACH WITH THE NCC}

The National Combustion Code (NCC) was used to perform simulations of several Woodward, FST, Inc., LDI-3 injector configurations. NCC is a state-of-the-art computational tool that is capable of solving the time-dependent, Navier-Stokes equations with chemical reactions. NCC is being developed primarily at the NASA Glenn in order to support combustion simulations for a wide range of applications, and has been extensively validated and tested for low-speed chemically reacting flows.

NCC uses second-order accurate central-differences for the convective and diffusion flux discretization, and a Jameson operator (a blend of 2 nd and 4th-order dissipation terms) for numerical stability. The second and fourth order dissipation parameters are typically set to $10^{-4}$ and 0.05 , respectively ([Swanson 1997]). The value of $k_{2}$, the constant that scales the second order dissipation gradient switch, is typically set to 0.25 . In order to enhance convergence acceleration in pseudo-time, implicit residual smoothing is used to smooth the computed residuals in NCC RANS simulations. Turbulence closure is obtained by using a two-equation, cubic k- $\varepsilon$ model with variable $C \mu$ ([Shih 1998]) and dynamic wall functions with pressure gradient effects ([Shih 2000]).

\subsection{LDI-3 GEOMETRY, MESHING AND CFD SETUP}

The National Combustor Code (NCC) was used to perform simulations of several multiple element WFST LDI-2 injector configurations comprised of various central pilot-injector and four surrounding maininjection elements (see figure 1). A candidate LDI-3 arrangement with a minimally recessed pilot injector was designated as the 'baseline' configuration for the CFD-led injector optimization studies reported in this paper. All four main injectors have their venturi-exit plane in-line with combustor dump-plane.

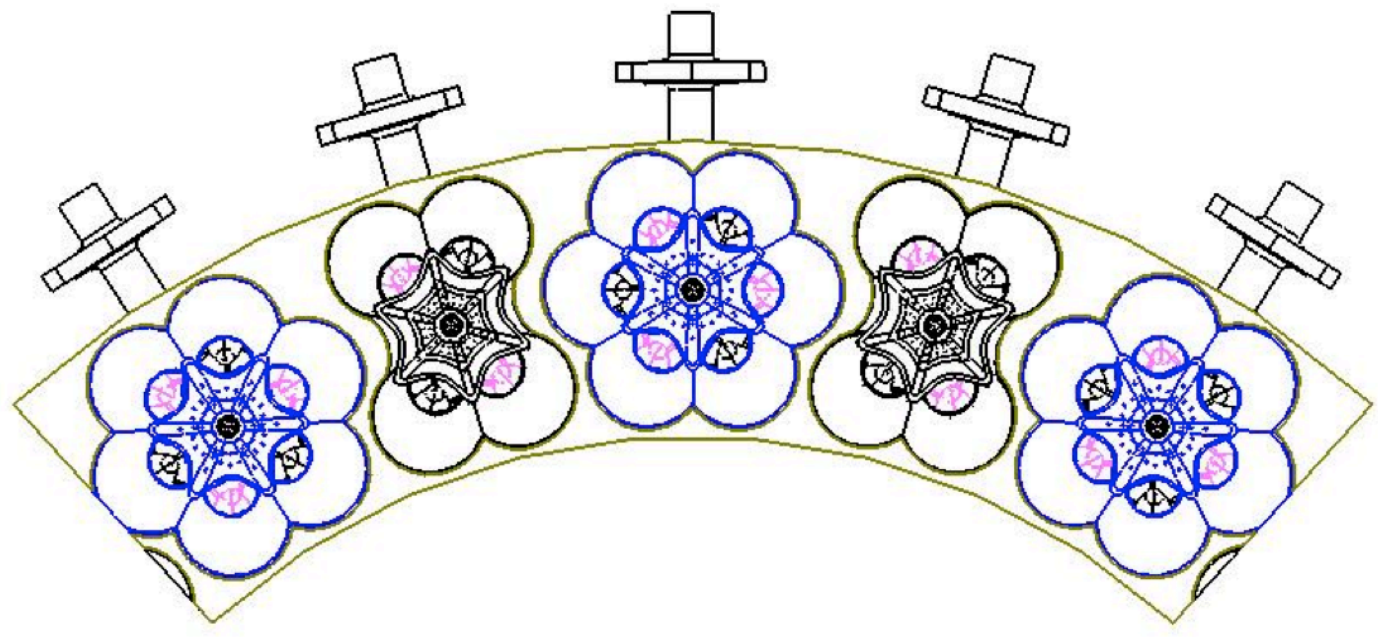

Figure 1. Five-sector dome-layout for a proposed LDI-3 injector configuration

The five-injection element sector of the five-sector LDI-3 geometry (see figure 1) was imported into the CUBIT mesh-generation software, to create a fully tetrahedral mesh with $9 \mathrm{M}$ to $11 \mathrm{M}$ elements, depending on the particular geometric configuration. Each blade passage and venturi was meshed as an individual block, and these blocks were then "imprinted" or merged with connecting volumes at their respective common surfaces. This ensured consistency of meshing across similar geometric elements, and also allowed for 'drop-in' replacement of the axial air swirlers for the main injectors, and/or the radial airswirler for the pilot, without requiring complete mesh regeneration for the entire configuration with attendant potential inconsistency. These best-practices for mesh generation established in earlier LDI-1 work [Ajmani 2013] with NCC were successfully used in CFD analysis of LDI-2 injector configurations [Ajmani 2014a]. 


\subsection{PARAMETRIC VARIATIONS FOR LDI-3 GEOMETRY}

Only the following two configurations out of the various parametric variations investigated with NCC for the LDI-3 injectors are summarized below:

1. Pilot Injector with axial fuel-injection - Integrated multi-element injector with circumferential slots for air injection varied the pilot air swirler offset inlets by $20 \%, 30 \%$ and $45 \%$ with attendant change in its swirl strength.

2. Main Injectors with discrete radial fuel injection points: Controlled confined flow established by $45^{\circ}$ or $52^{\circ}$ axial bladed swirl vanes, rotating clockwise $(\mathrm{CW})$ or counter-clockwise $(\mathrm{CCW})$ relative to the pilot injector, in addition to three different venturi-lengths.

\subsection{PARAMETRIC DESIGN OF RADIAL INJECTION MODULE WITH THE NCC}

NCC RANS Computations of non-reacting flow were performed for several different parametric setups as described in section 3.2. As shown in figure 2, a fully tetrahedral mesh consists of approximately $9 \mathrm{M}$ to $11 \mathrm{M}$ elements was generated for a five-injector geometry (one of the five sectors of the five-sector dome layout shown in figure 1). Best-practices for meshing of LDI-1 injector elements reported in [Ajmani 2013] were leveraged for LDI-3 meshing.
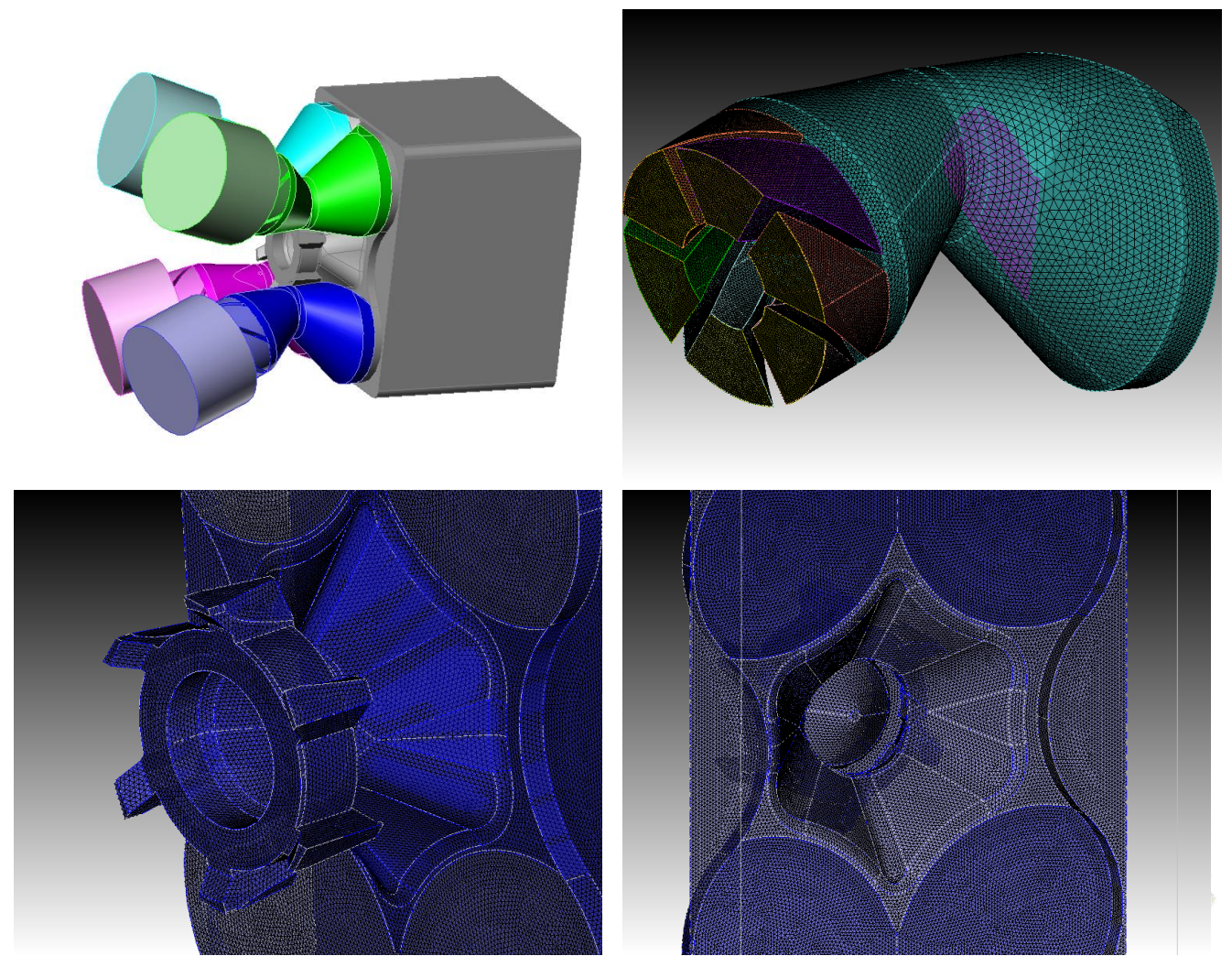

Figure 2. Geometry for CFD Analysis (top left) and Surface Meshes for Main Injector (top right) and Pilot Injector (bottom left, bottom right))

- The following boundary conditions were used:

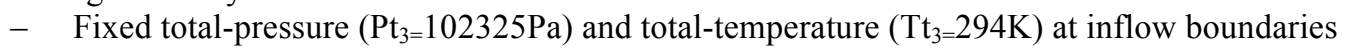

- Fixed static-pressure $\left(\mathrm{P}_{4}=\mathrm{P}_{3}-\Delta \mathrm{P}\right)$ at outflow $(\Delta \mathrm{P}=1000 \mathrm{~Pa})$

- Adiabatic, no-slip conditions at all walls 
Each non-reacting flow simulation consisted of NCC RANS computations till a 'mass-imbalance (outflow-inflow) convergence of $0.1 \%$ over 500 consecutive NCC RANS iterations was achieved. For a typical case, 100,000 RANS iterations at a CFL of 1.95 were run, to obtain a converged, steady-state, nonreacting flowfield. The CFD predicted mass flow rate at the inlet of each injector was used to determine the $\mathrm{AC}_{\mathrm{d}}$ of each individual injector, and the total $\mathrm{AC}_{\mathrm{d}}$ of the five-injector configuration was compared to measured values of $\mathrm{AC}_{\mathrm{d}}$ provided by WFST. The goal of the parametric study reported here was to determine the optimal configuration that would satisfy the following requirements:

1. maximize the total $\mathrm{AC}_{\mathrm{d}}$ of the five-element array

2. minimize the near-wall flow separation downstream of the throat section (main injectors)

3. minimize the venturi length while meeting criteria 1 and 2

4. provide an 'optimal' recirculation zone of approximately 1" length, downstream of the pilot

\subsection{CFD Results: Effect of varying Pilot Offset on $\mathrm{AC}_{\mathrm{d}}$ and flow characteristics}

NCC RANS computations were performed on three different configurations of the pilot injector with $45^{\circ}$ axially bladed swirlers for the four main injectors. The "percentage offset" of the six air-injection slots (see figure 3) is designed to control the amount of swirl in the pilot chamber, and hence influence the size of the primary recirculation zone in the main combustor. A CFD analysis was performed by reducing the amount of the air-flow slot offset from a nominal value of $45 \%$ to $30 \%$ and $20 \%$, respectively.

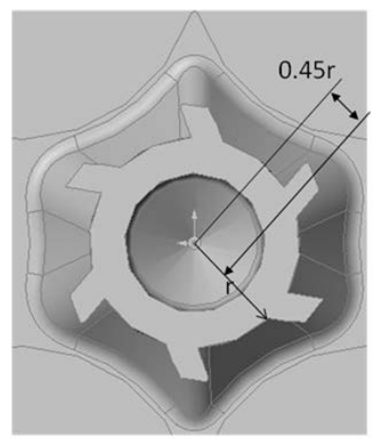

$45 \%$ Offset

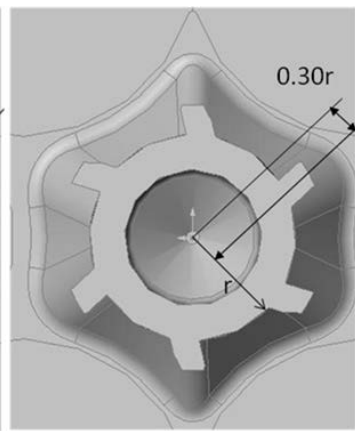

$30 \%$ Offset

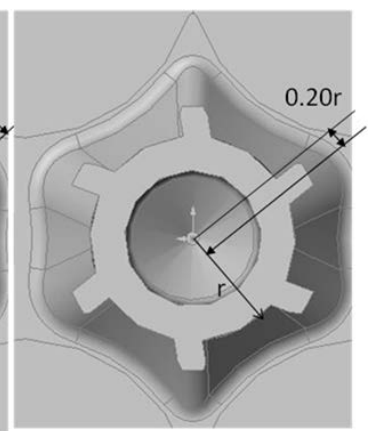

$20 \%$ Offset

Figure 3. Parametric variation for Pilot Injector - variation of offset value of air-flow slots.

The effect of reducing the slot offset of the pilot injector airflow inlets on the $\mathrm{AC}_{\mathrm{d}}$ of the pilot injector is summarized in Table 1 . There is excellent agreement (1\% error) in the CFD prediction of total effective area for the $45 \%$ offset case, when compared with the measured effective area. The CFD predicts that the total effective area of the five-injector array changes marginally from 0.587 to 0.577 , a $1.7 \%$ reduction when reducing the pilot offset from $45 \%$ to $20 \%$. As expected, even a major change in the pilot offset angle does not have a significant effect on total effective area. However, the individual effective area of the pilot injector does increase by $5 \%$ when the offset is reduced from $45 \%$ to $20 \%$. This is due to the reduced swirl in the $20 \%$ offset case, which in turn reduces the pressure loss through the pilot injector.

Table 1. Effect of changing the offset percentage of pilot airflow slots on effective area $\left(A_{d}\right)$

\begin{tabular}{|c|c|c|c|}
\hline Effective Area $\left(A C_{d}\right)$ & Total $\left(\right.$ in $\left.^{2}\right)$ & Mains (in ${ }^{2}$ ) & Pilot $\left(\right.$ in $\left.^{2}\right)$ \\
\hline Measured $45^{\circ} \mathrm{AS}$ (45\% Offset) & 0.592 & 0.527 & 0.065 \\
\hline NCC CFD $45^{\circ}$ AS (45\% Offset) & 0.587 & 0.5226 & 0.0644 \\
\hline NCC CFD $45^{\circ}$ AS (20\% Offset) & 0.577 & 0.5085 & 0.0685 \\
\hline
\end{tabular}

$\mathrm{AC}_{\mathrm{d}}$ for Pilot, Mains (CFD) $=\mathrm{mdot}_{\mathrm{CFD}} / \mathrm{sqrt}(2 * \Delta \mathrm{P} *$ rho $) \quad\left(\mathrm{CFD}\right.$ uses imposed value of $\Delta \mathrm{P}=1000 \mathrm{~Pa}$ for $\mathrm{AC}_{\mathrm{d}}$ computation)

Table 1 shows that the predicted effective area of the four main injectors is also influenced by the amount of offset of the pilot airflow slots. At $20 \%$ offset, the main injectors suffer a $2.7 \%$ reduction in effective area, as compared to that for $45 \%$ pilot offset. An examination of the axial velocity contours through the pilot and main injectors (see figure 4) shows that the size of the pilot recirculation zone is 
marginally smaller for the $20 \%$ offset case, which changes the back-pressure 'seen' by the main injectors, and hence reduces the effective area for the mains. The axial-velocity at the combustor dump plane (namely at $x=0.0 \mathrm{~mm}$ location in figure 5) shows much greater non-uniformity in the central pilot cup for the $20 \%$ offset configuration. In addition, the flow within the pilot cup suffers near-wall separation upstream of the dump plane (see $x=-5 \mathrm{~mm}$ location). These flow non-uniformities for the $20 \%$ pilot offset, combined with the greater total effective area with the $45 \%$ pilot offset, identified the $45 \%$ pilot offset configuration as the preferred selection of this parametric study. The next steps of the CFD parametric study with NCC focused on optimizing the flow and effective area of the main injectors.

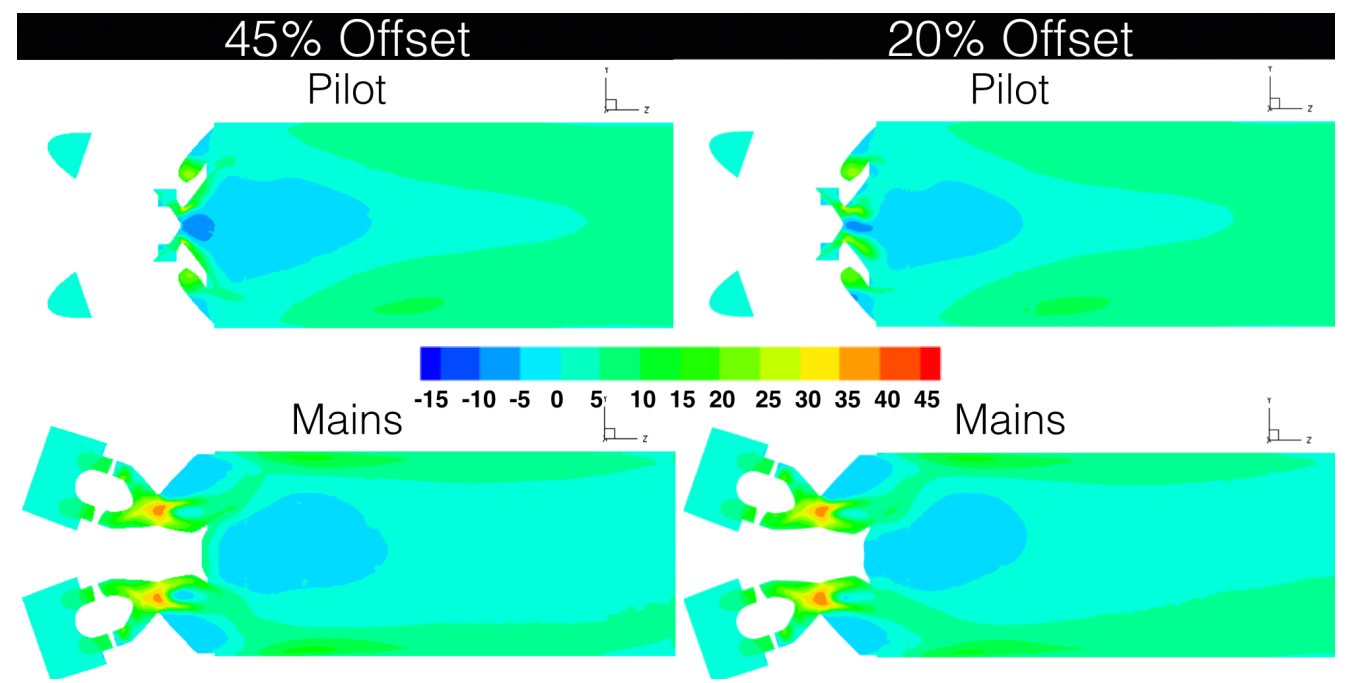

Figure $4.45 \%$ vs. $20 \%$ Pilot Injector Offset - Axial Velocity contours $(\mathrm{m} / \mathrm{s})$ in two cross-sectional planes (Pilot at top, Mains at bottom)

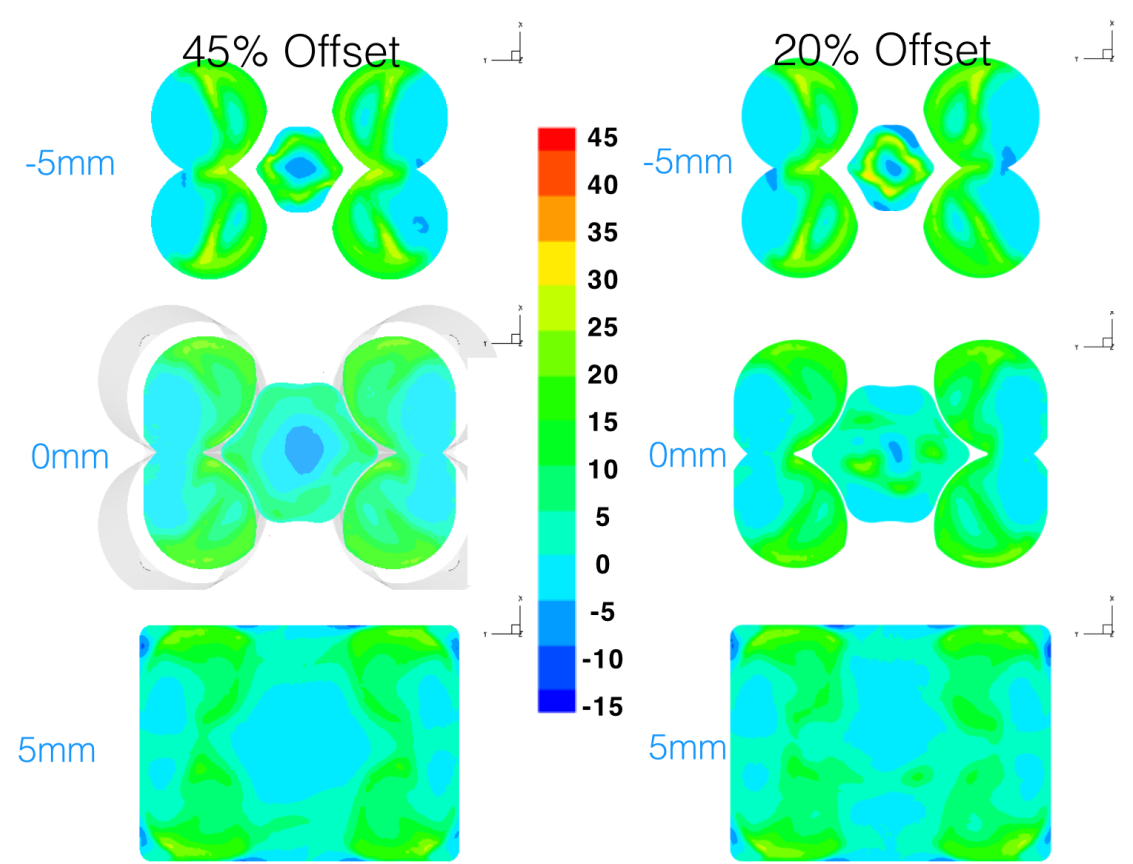

Figure 5. $45 \%$ vs. $20 \%$ Pilot Injector Offset - Axial Velocity contours $(\min =-15, \max =45 \mathrm{~m} / \mathrm{s})$ in three axial cross-sections (dump plane at $\mathrm{x}=0.0 \mathrm{~mm}$ ) 


\subsection{CFD Results: Effect of varying Swirler Blade Angle on $\mathrm{AC}_{\mathrm{d}}$ and flow characteristics}

The effect of changing the turning angle in the six vane passages of the axial-bladed venturis on the effective area and flowfield of the five-injector array was studied with NCC RANS simulations. Higher swirler angles in the axial-bladed venturis tend to produce larger central recirculation zones [Fu 2008], which can help with flame stability and operability at some engine power conditions. However, for a given equivalence ratio, injectors with higher swirler angles do increase NOx emissions [Tacina 2005]. In addition, higher swirler angles increase the viscous losses through the blade passages, which decreases the effective area of the injector. The current CFD analysis predicts that an increase in swirler angle from $45^{\circ}$ to $52^{\circ}$ would lead to a $7.5 \%$ reduction in effective area, i.e. from $0.587 \mathrm{in}^{2}$ to $0.539 \mathrm{in}^{2}$ (see Table 2). The next step in the parametric study was to attempt to 'recover' this decrease in $\mathrm{AC}_{\mathrm{d}}$ by varying the length of the venturi section downstream of the throat.

A comparison of the axial velocity contours for the two swirler angles is shown in figures 6 and 7. Two major changes are introduced in the flowfield as a result of the switch from $45^{\circ}$ swirler to $52^{\circ}$ swirler angle. The central recirculation zone behind the pilot is smaller for the $52^{\circ}$ swirler as compared to the $45^{\circ}$ swirler. This observation has to be viewed in the context of the flowfield produced by the four neighboring main injectors. At the higher swirler angle in the main injectors, the near-wall corner separation zone disappears, and is replaced by a central recirculation zone. This is a desirable outcome of the switch to the $52^{\circ}$ swirler, from the perspective of flame holding in the center of the venturi rather than at the venturi wall. The next two sections report the CFD analysis of varying other geometric parameters of the main injector to compensate for the loss in effective area due to the switch to $52^{\circ}$ swirlers.

Table 2. Effect of changing the swirler blade angle on effective area $\left(\mathrm{AC}_{\mathrm{d}}\right)$

\begin{tabular}{|c|c|c|c|}
\hline Effective Area $\left(\mathrm{AC}_{\mathrm{d}}\right)$ & Total $\left(\mathrm{in}^{2}\right)$ & Mains (in $\left.{ }^{2}\right)$ & Pilot (in $\left.{ }^{2}\right)$ \\
\hline Measured $45^{\circ} \mathrm{AS}$ (45\% Offset) & 0.592 & 0.527 & 0.065 \\
\hline NCC CFD1A $45^{\circ}$ AS (45\% Offset) & 0.587 & 0.523 & 0.064 \\
\hline NCC CFD2C $52^{\circ} \mathrm{AS}(45 \%$ Offset) & 0.539 & 0.477 & 0.062 \\
\hline
\end{tabular}

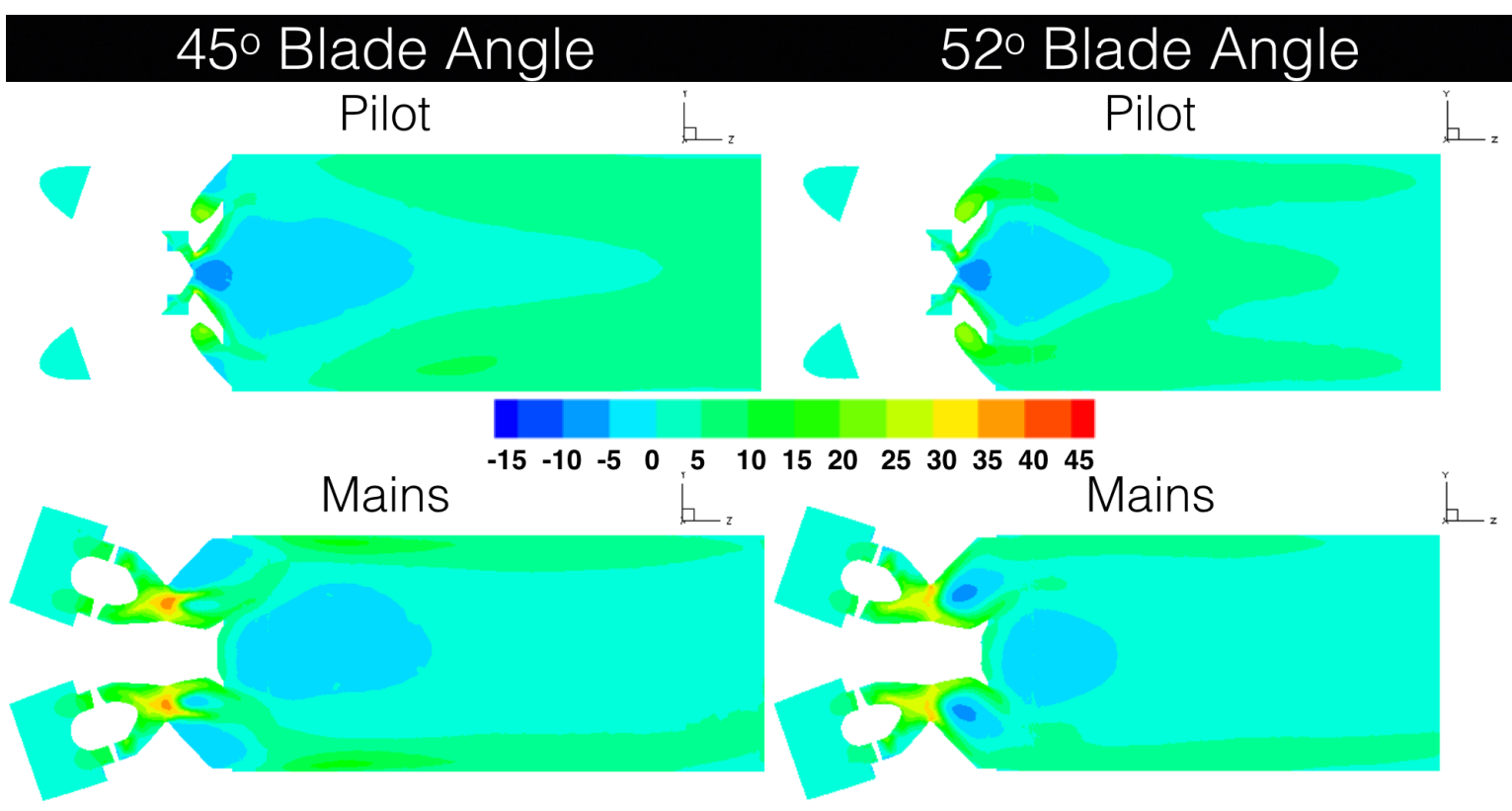

Figure 6. $45^{\circ}$ vs. $52^{\circ}$ Swirler Blade Angle - Axial Velocity contours $(\mathrm{m} / \mathrm{s})$ in two cross-sectional planes (Pilot at top, Mains at bottom) 

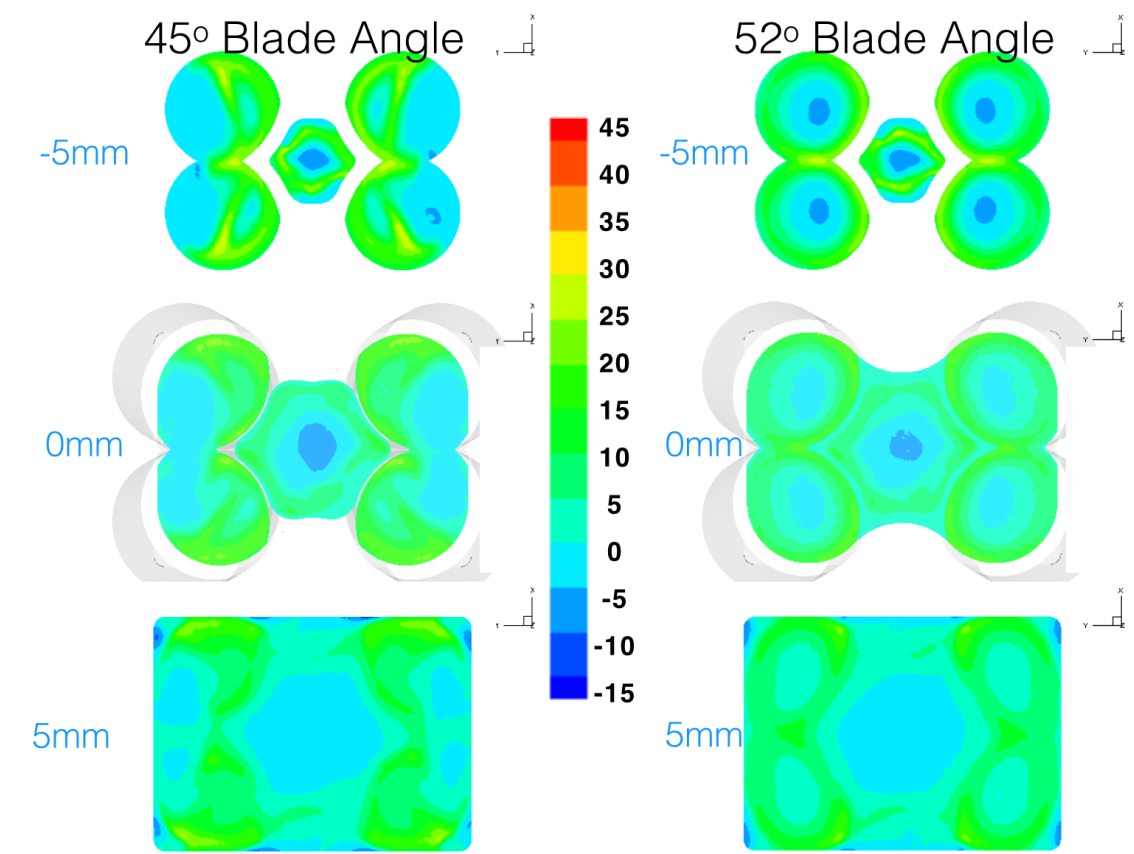

Figure 7. $45^{\circ}$ vs. $52^{\circ}$ Swirler Blade Angle - Axial Velocity contours (m/s) in three axial cross-sections (dump plane at $\mathrm{x}=0.0 \mathrm{~mm}$ )

\subsection{CFD Results: Effect of varying Venturi Length on $\mathrm{AC}_{\mathrm{d}}$ and flow characteristics}

The effect of changing the venturi length downstream of the throat section of the four main injectors on the effective area and flowfield of the five-injector array was studied with NCC RANS simulations. From a combustor sizing and weight perspective, it is desirable to have the shortest feasible total injector length that will meet the combustor performance and stability requirements. Table 3 shows that a decrease in venturi length from 0.55 " to 0.47 " increases the $\mathrm{AC}_{\mathrm{d}}$ from $0.470 \mathrm{in}^{2}$ to $0.539 \mathrm{in}^{2}$, a $15 \%$ increase. However, as will be verified in the next section, the primary reason for this increase in $\mathrm{AC}_{\mathrm{d}}$ is due to the concurrent decrease in hub diameter, which provides for greater open area in the blade passages.

Table 3. Effect of changing the venturi length on effective area $\left(A C_{d}\right)$

\begin{tabular}{|c|c|c|c|}
\hline Effective Area $\left(\mathrm{AC}_{\mathrm{d}}\right)$ & Total $\left(\right.$ in $\left.^{2}\right)$ & Mains (in $\left.{ }^{2}\right)$ & Pilot (in $\left.{ }^{2}\right)$ \\
\hline Measured ( $52^{\circ} \mathrm{AS}$, Medium Venturi $\left.0.55^{\prime \prime}\right)$ & 0.439 & 0.373 & 0.066 \\
\hline NCC CFD2A (52 ${ }^{\circ} \mathrm{AS}$, Medium Venturi $\left.0.55^{\prime \prime}, 0.5^{\prime \prime} \mathrm{Hub}\right)$ & 0.470 & 0.402 & 0.068 \\
\hline NCC CFD2C (52 AS, Short Venturi 0.47”, 0.4” Hub) & 0.539 & 0.477 & 0.062 \\
\hline
\end{tabular}

The major flow effect of decreasing the venturi length is observed in the shift of the central recirculation (medium venturi) to a large "corner" separation (short venturi), in the main injector venturis downstream of the throat (figures 8,9). In addition, the short venturi produces a much larger central recirculation zone behind the pilot injector, which is due to the change in the flow exiting the main injectors in the vicinity of the pilot venturi. The corner recirculation flow pattern in the short venturi was considered unacceptable, and a medium venturi configuration with a smaller (0.4") hub size was analyzed to increase the effective area. 


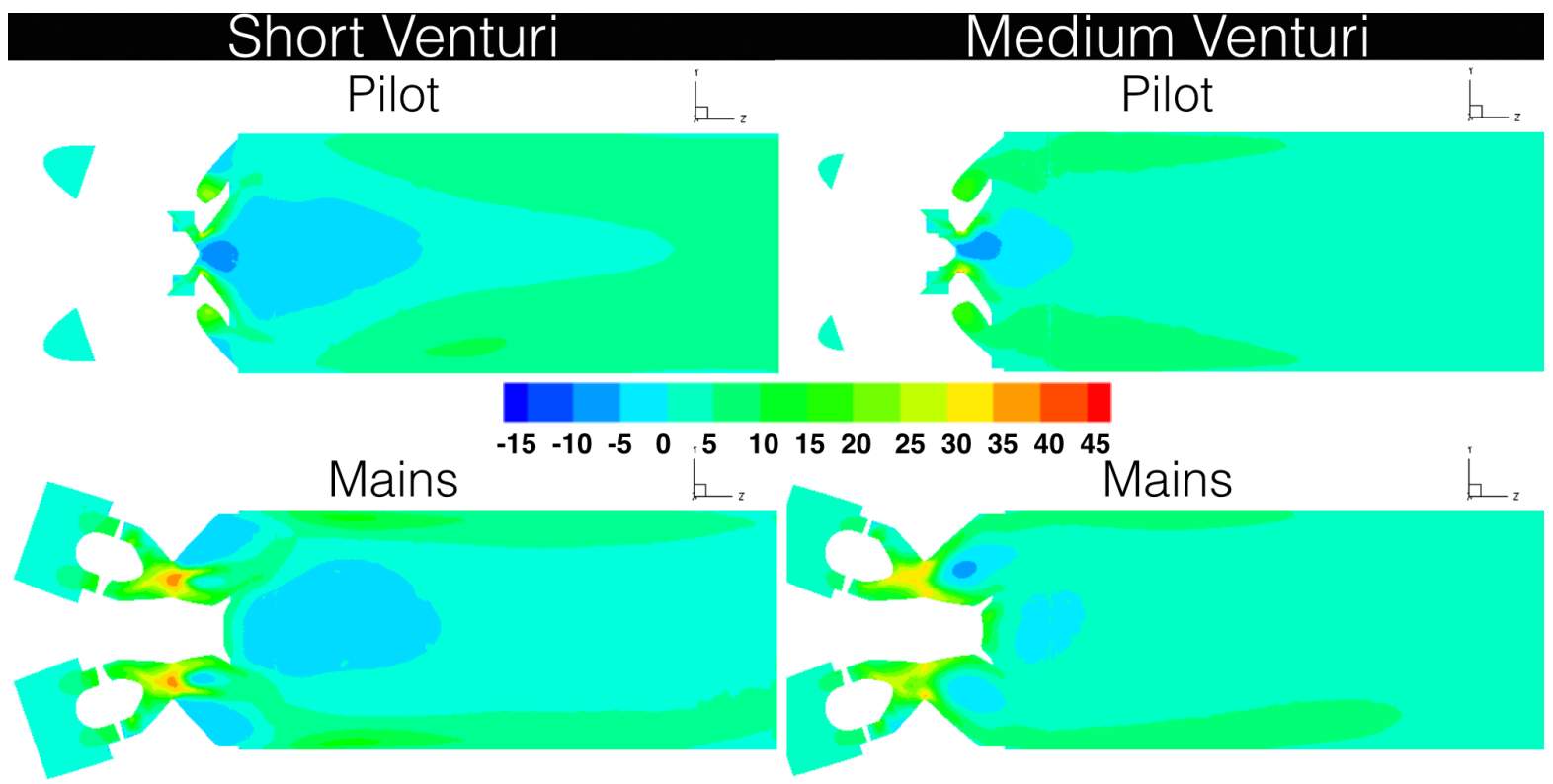

Figure 8. Short vs. Medium Venturi Length - Axial Velocity contours $(\mathrm{m} / \mathrm{s})$ in two cross-sectional planes (Pilot, Mains)

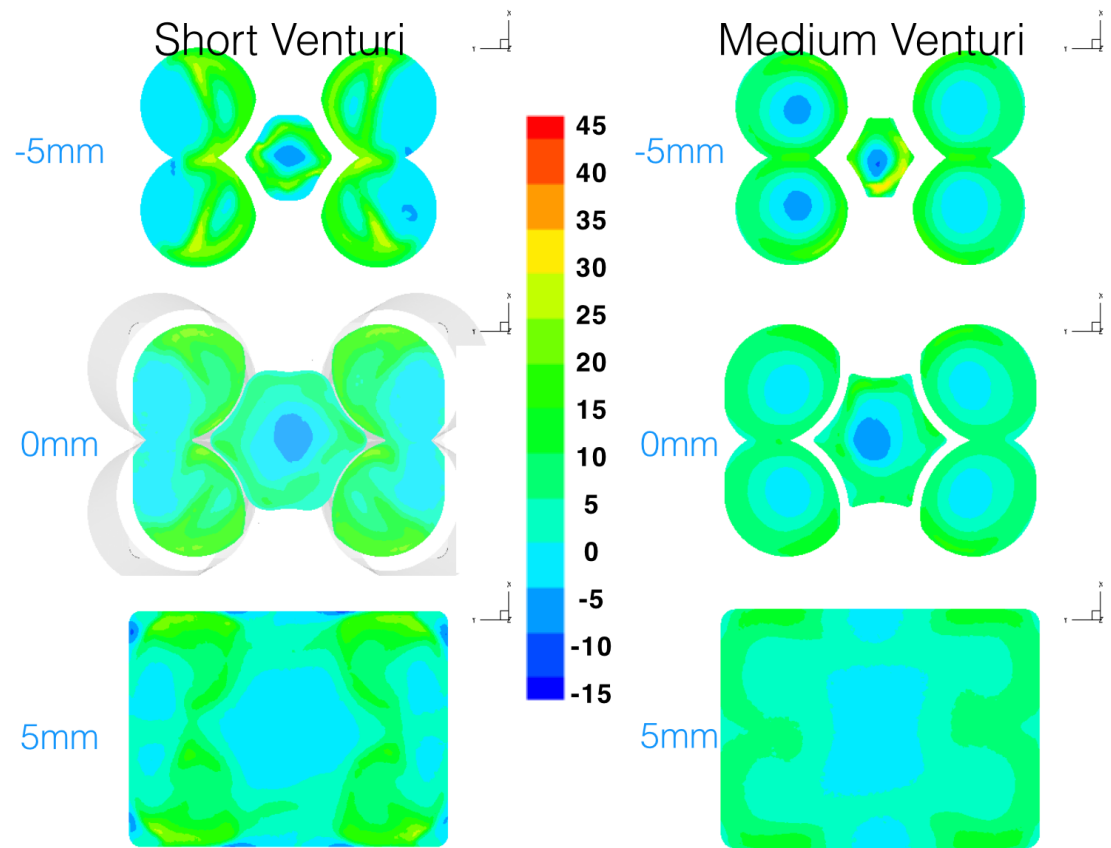

Figure 9. Short vs. Medium Venturi Length - Axial Velocity contours $(\mathrm{m} / \mathrm{s})$ in three axial cross-sections (dump plane at $\mathrm{x}=0.0 \mathrm{~mm}$ )

\subsection{Effect of varying $\mathrm{Hub}$ Diameter on $\mathrm{AC}_{\mathrm{d}}$ and flow characteristics}

The final geometry parametric studied with CFD analysis was the effect of hub diameter of the six blade passages in each of the four main injectors on the effective area and flowfield of the five-injector array. This additional parametrer was included in the CFD study in order to attempt to 'recover' the reduction in $\mathrm{AC}_{\mathrm{d}}$ caused by higher swirler angle $\left(45^{\circ}\right.$ to $\left.52^{\circ}\right)$ and longer venturi length (0.47" to $\left.0.55^{\prime \prime}\right)$. A summary of the various configurations with different hub-sizes is shown in Table 4. For the medium venturi length, a decrease in hub size from 0.5 " to 0.4 " increases the $\mathrm{AC}_{\mathrm{d}}$ from $0.47 \mathrm{in}^{2}$ to $0.559 \mathrm{in}^{2}$, a $19 \%$ 
increase. For the short venturi length, a decrease in hub size from 0.4 " to 0.35 " increases the $\mathrm{AC}_{\mathrm{d}}$ from $0.539 \mathrm{in}^{2}$ to $0.628 \mathrm{in}^{2}$, a $19 \%$ increase.

Table 4. Effect of changing the hub diameter of axial-bladed swirlers on effective area $\left(\mathrm{AC}_{\mathrm{d}}\right)$

\begin{tabular}{|c|c|c|c|}
\hline Effective Area $\left(\mathrm{AC}_{\mathrm{d}}\right)$ & Total (in $\left.{ }^{2}\right)$ & Mains (in $\left.{ }^{2}\right)$ & Pilot (in $\left.{ }^{2}\right)$ \\
\hline Measured $\left(45^{\circ} \mathrm{AS}\right.$, Short Venturi, $0.5^{\prime \prime}$ Nominal Hub) & 0.592 & 0.527 & 0.065 \\
\hline NCC CFD2A (52 ${ }^{\circ} \mathrm{AS}$, Medium Venturi, 0.5" Nominal Hub) & 0.470 & 0.402 & 0.068 \\
\hline NCC CFD3A (52 ${ }^{\circ} \mathrm{AS}$, Medium Venturi, 0.4" Small Hub) & 0.559 & 0.492 & 0.067 \\
\hline NCC CFD2C (52 AS, Short Venturi, 0.4" Small Hub) & 0.539 & 0.477 & 0.062 \\
\hline NCC CFD2D (52 ${ }^{\circ} \mathrm{AS}$, Short Venturi, 0.35” Extra Small Hub) & 0.628 & 0.563 & 0.065 \\
\hline
\end{tabular}

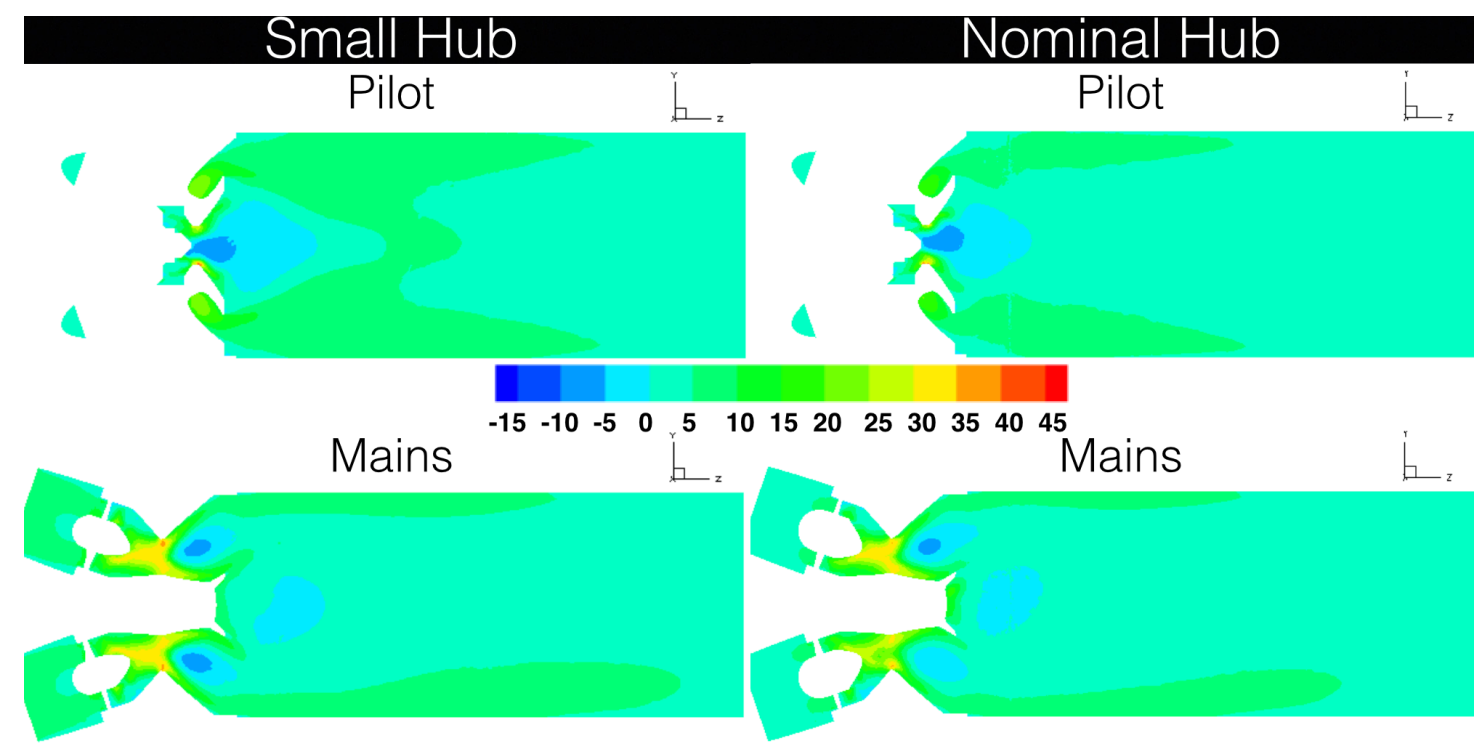

Figure 10. Small (0.5") vs Nominal (0.4") Hub Diameter - Axial Velocity contours (m/s) in two crosssectional planes (Pilot, Mains)

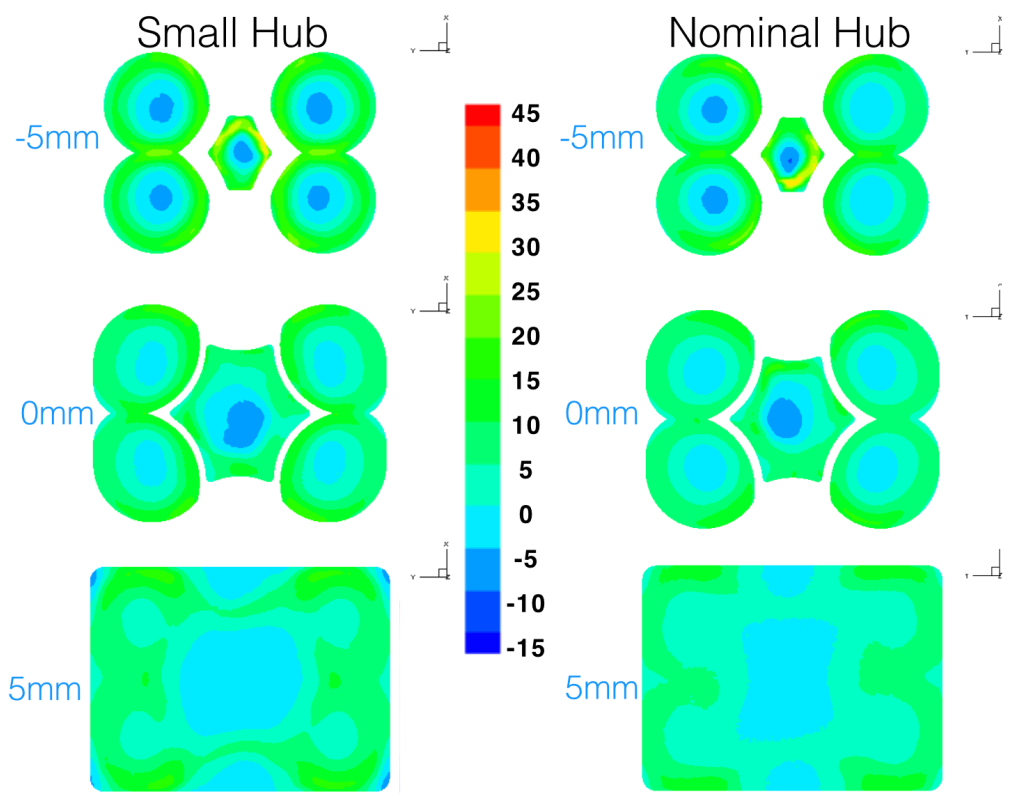

Figure 11. Small (0.5") vs Nominal (0.4") Hub Diameter - Axial Velocity contours (m/s) in three axial cross-sections (dump plane at $\mathrm{x}=0.0 \mathrm{~mm}$ ) 
As shown earlier in figures 8 and 9, the combination of a short venturi with small hub (0.4") produces undesirable flow features (corner separation in mains, large central recirculation behind pilot). The short venturi with an extra-small hub (0.35") shows similar flow patterns (not shown here). For a medium length venturi, figures 10 and 11 show the effect of decreasing the hub diameter from nominal (0.5") to small value of 0.4 ". The flow remains largely unchanged by the change in the hub diameter, both in the pilot and mains. This is because the geometry change introduced by the hub diameter variation is far enough upstream of the venturi throat, so as to cause minimal change in the flow downstream of the throat.

In summary, the combination of $52^{\circ}$ axial swirler vane angle, medium length ( $\left.0.55^{\prime \prime}\right)$ venturi and small (0.4") hub produce acceptable flow features in the main venturis and behind the pilot injector without sacrificing the effective area. Hence, this configuration selected as the optimized design for conducting detailed, reacting flow CFD analysis described in the next section.

\subsection{Single Injector Element Reacting Flow Optimization}

The insights obtained from the CFD results for the parametric study performed on the various configurations of the LDI-3 injector array with NCC RANS were used to arrive at an "optimized configuration" for the purpose of manufacturing and preliminary testing with spray injection and reacting flow. The optimized configuration includes $30 \%$ pilot Offset, $52^{\circ}$ air-swirler angle, short venturi length (0.47"), and a 0.4 " hub diameter. Four rows of holes were added to the venturi surface to represent the cooling flow to be injected downstream of the throat in the injector. These cooling holes had not been meshed in the nonreacting injector optimization study described in previous sections, in order to simplify the mesh generation process. The geometry was meshed with the CUBIT software, and the final mesh consists of approximately 5M tetrahedral elements. Details of the optimized final geometry and the surfacemesh for the main injector are shown in figure 12.

Non-reacting flow computations with NCC RANS were performed for the optimized single-injector to determine the effective area and pressure drop of this configuration. The boundary conditions and convergence criteria used for the parametric design (see section 4) were also used for this analysis. At atmospheric conditions, the NCC prediction of total effective area was $0.119 \mathrm{in}^{2}$, which is $8 \%$ lower than the measured effective area of $0.13 \mathrm{in}^{2}$. NCC predicted that $14 \%$ of the total airflow is drawn through the three rows of cooling holes, which agrees very well with the $15 \%$ estimate provided by WFST. Note that one of the four cooling rows was blocked off in the final CFD computations in order to reduce the cooling flow to $15 \%$ of total air flow. The converged, non-reacting flow solution was used to initialize NCC reacting-flow computations with liquid fuel (Jet-A) spray injection, ignition and finite-rate kinetics modeling. A 14-species, 18-step chemical kinetics model for Jet-A/Air combustion, designed and validated for LDI combustion systems [Ajmani 2014c] was used to model the reacting flow.

As shown in figure 12, the fuel flow is injected slightly upstream of the throat, and normal to the venturi surface. The reacting flow CFD computations with NCC RANS simulate the spray with a Lagrangian, particle-tracking formulation [Raju 2012]. Based on experimental observations of spray injection with a pressure-atomizer at WFST, a $60^{\circ}$ hollow cone spray with annular angle width of $10^{\circ}$ (viz. droplets spread within $55^{\circ}$ to $65^{\circ}$ region) was modeled with NCC. At each spray time-step, the spatial streams were permitted a stochastic variation of the stream location within the $10^{\circ}$ cone thickness. As the typical SMD $\left(\mathrm{d}_{32}\right.$ provided by Woodward FST) for the conditions computed here was less than $10 \mu \mathrm{m}$, the droplets are assumed to undergo evaporation without any primary or secondary breakup.

The Lagrangian solution process used for this study employs an unsteady spray model such that droplet groups are only integrated for a fraction of their lifetime (but restarted at this point for the next iteration), rather than to a completely steady-state solution. The typical spray integration time-steps were $2 \mathrm{e}-7 \mathrm{~s}$ (local time-step) and 4e-5s (global time-step), which translates to 200 local time-steps for each global time-step for the spray solver. An inflow droplet size distribution is prescribed by the correlation equation: 


$$
\frac{d n}{n}=4.21 \times 10^{6}\left[\frac{d}{d_{32}}\right]^{3.5} e^{-16.98\left(\frac{d}{d_{32}}\right)^{0.4}} \frac{d d}{d_{32}}
$$

Here $n$ is the total number of droplets, $d_{32}$ is the Sauter mean diameter (SMD), and $d n$ is the number of droplets in the size range between $d$ and $d+d d$. In this work, ten 'droplet groups' are used to represent the drop size distribution among a finite number of droplet classes.

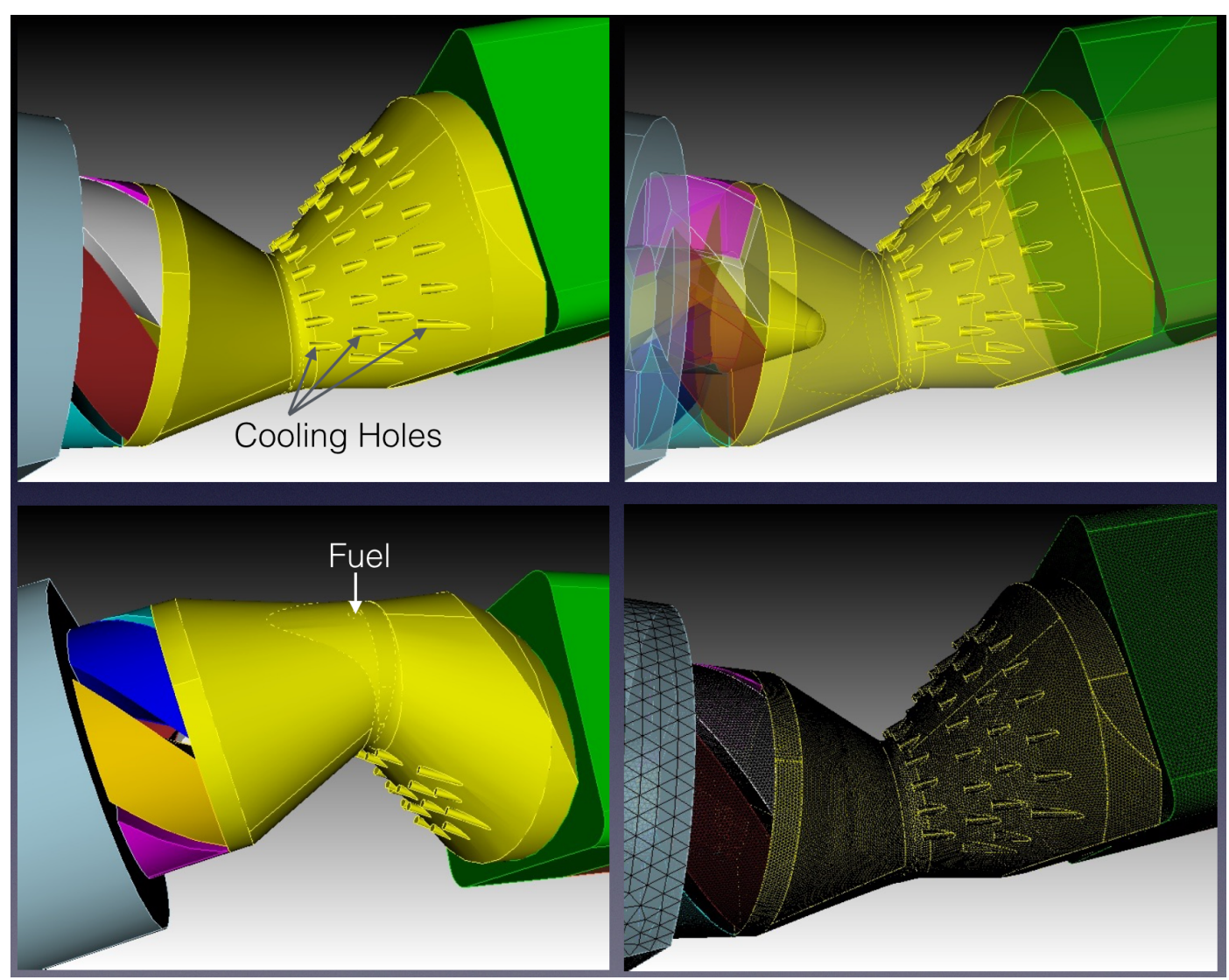

Figure 12. Optimized Single Injector with cooling holes: geometry and surface-mesh

The fuel droplet distribution and temperature contours predicted by NCC for the single element configuration at typical 'medium power' cycle conditions $\left(\mathrm{P}_{3}=1.75 \mathrm{MPa}, \mathrm{T}_{3}=810 \mathrm{~K}, \Delta \mathrm{p}=4 \%, \mathrm{FAR}=0.0214\right)$ are shown in figure 13. The fuel droplets, injected radially near the throat, seem to be rapidly transported towards the walls of the injector by the airflow in the venturi. This results in a very irregular and unstable mixing of fuel and air, which produces streaks of alternating high and low temperature regions in the injector venturi and the combustor. The overall nature of the fuel distribution pattern remained largely unchanged when the CFD analysis was repeated by moving the fuel injection either axially upstream, circumferentially along the throat, and radially towards the injector centerline. The aerodynamics within the injector seems to largely dominate the fuel distribution pattern, regardless of the injection location of the fuel.

Figure 14 shows the contours of mean axial, radial and tangential velocity components within the injector along the centerlines of the $\mathrm{x}$-axis (X-MID) and $\mathrm{y}$-axis (Y-MID). Note that the centerline of the spray injection is in the X-MID plane, slightly upstream of the throat. The axial velocity contours show the presence of a significant central recirculation zone in the venturi, downstream of the throat section. Some very weak near-wall separation is observed in the blade passages, and mild separation is observed at the bottom wall of the injector downstream of the dump plane where the lip of the pilot injector intrudes into the main injector. There are fairly thick boundary layers immediately downstream of the throat, and the peak axial velocity near the throat is $45 \mathrm{~m} / \mathrm{s}$. 
The CFD results predict that the fuel would encounter fairly strong circumferential momentum and minimal radial momentum, when introduced at the specified wall location upstream of the throat. Note the thickness of the circumferential velocity boundary layer near the injection location in the radial direction, and its extent all along the lower wall of the venturi (dark blue region in circumferential velocity X-Mid plot). This aerodynamic behavior partly explains why the predicted spray distribution pattern remained largely unchanged when the fuel injection location was moved axially upstream.

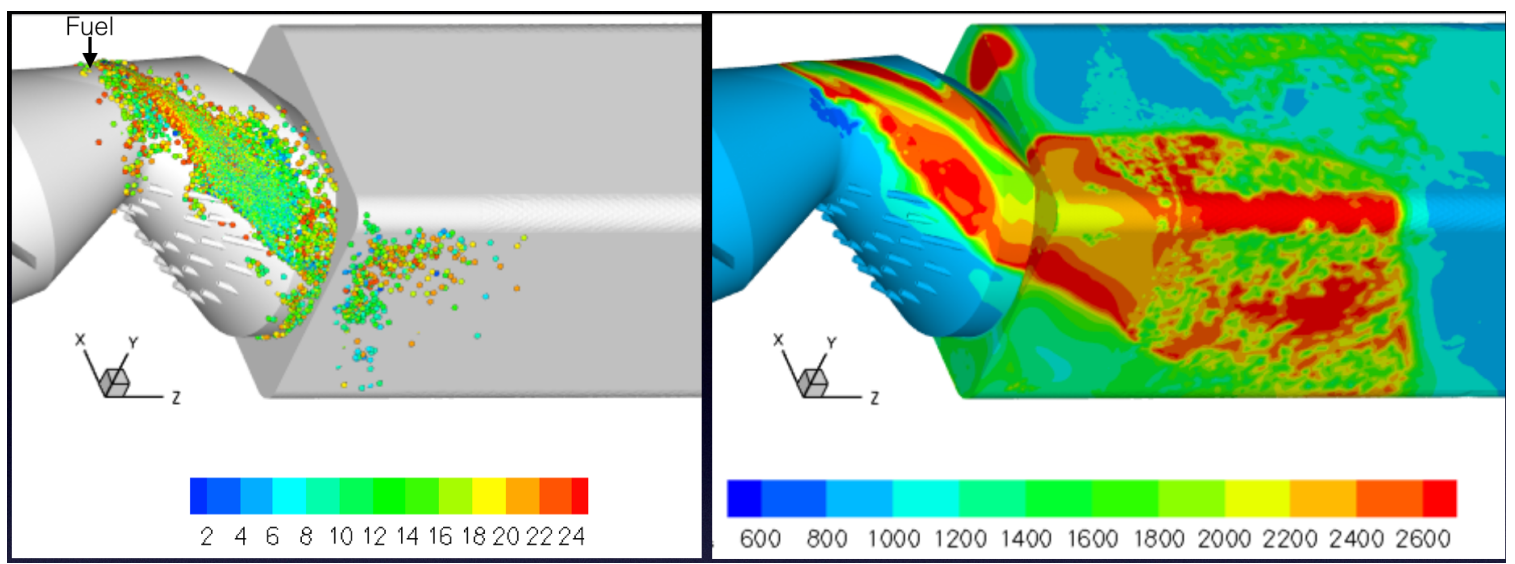

Figure 13. Single Injector with cooling holes: particle distribution (left), temperature (K) contours (right)

An alternate fuel injection location is perhaps at a station $\mathrm{A}$ in the X-Mid plane, or station B in the YMid plane. At either location, the weak circumferential velocity, combined with strong radial velocity would tend to push the fuel towards the center, to enable better penetration and mixing. The current layout of the integrated multi-point injector module, where all four main injectors would be fuel-fed by a single, central fuel supply module, makes either of these injection locations somewhat challenging. Future CFD analysis will focus on the effects of these proposed alternate injector locations to mitigate these challenges.

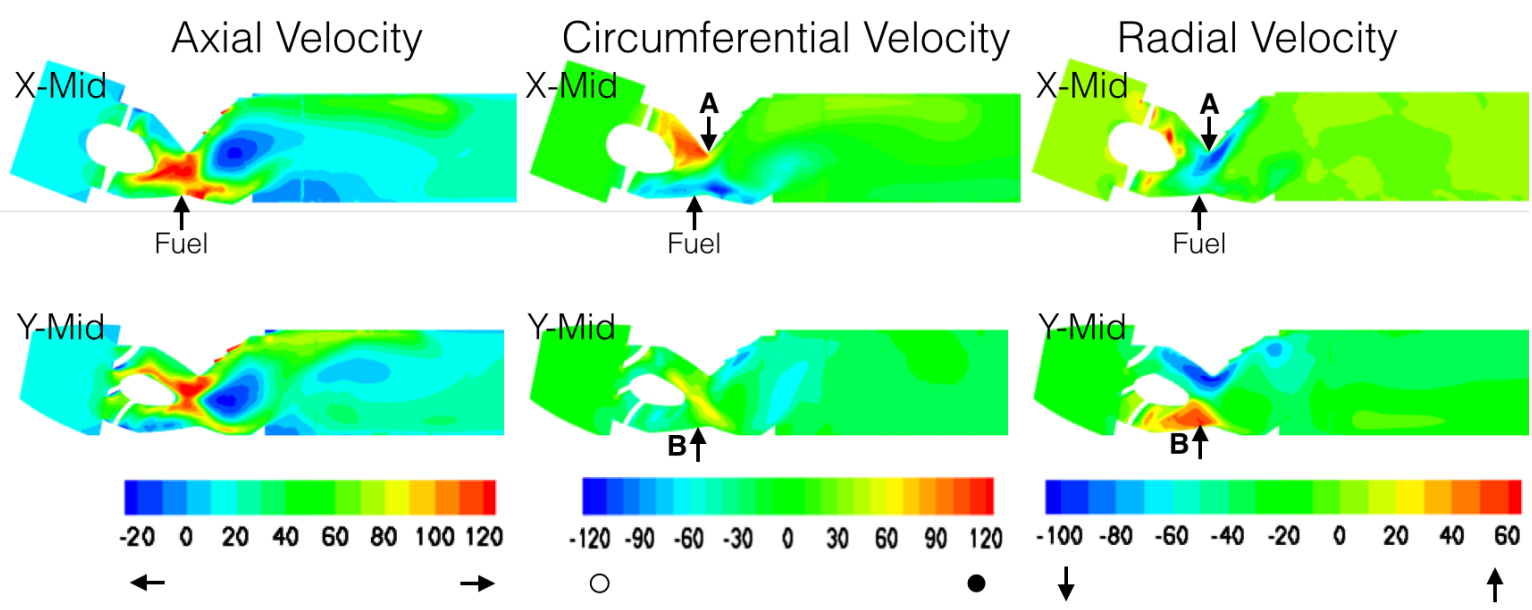

Figure 14. Predicted velocity components $(\mathrm{m} / \mathrm{s})$ at two axial planes of the single injector configuration with current injection location (marked 'Fuel') at 'medium power' conditions

\subsection{SUMMARY}

The National Combustor Code (NCC) was used to perform parametric studies of proposed LDI-3 injectors designed by Woodward, FST Inc. Several non-reacting flow RANS simulations of an LDI-3 configuration consisting of four main-injectors (axial air flow, radial fuel flow) and an integrated pilot injector (radial air flow, axial fuel flow) were performed. Geometry parametrics studied with NCC included changing the amount of swirl in the pilot injector, the angle of the axial-bladed swirl venturi cups, the 
venturi length and the hub diameter of the main injectors. The goal was to arrive at an optimized configuration that would meet the design requirements of maximum effective area, 'optimal' size of primary recirculation zone, minimal near-wall separation in the main injector venturi, and minimal venturi length. This optimal injector array configuration was evaluated for its effectiveness in providing adequate fuel-air mixing in the main injectors. Reacting flow CFD simulations with a finite-rate kinetics model and lagrangian-spray modeling (for the liquid phase droplets) showed very poor results for the fuel injection approach where fuel is introduced in the main injectors in a radial direction normal to the primary air flow direction. Based on the current CFD results, two alternate fuel injections locations were proposed to improve the spray distribution. Future CFD work with NCC will analyze the performance of the main fuel injectors for these alternate fuel injection locations.

\subsection{REFERENCES}

[Ajmani 2013] Ajmani, K., Mongia, H.C. and Lee, P., "CFD Best Practices to Predict NOx, CO and Lean Blowout for Combustor Design," ASME IGTI Paper GT2013-95669, ASME Turbo Expo 2013, San Antonio TX, June 2013.

[Ajmani 2014a] Ajmani, K., Mongia, H. C., and Lee, P., "CFD computations of emissions for LDI-2 combustors with simplex and airblast injectors", AIAA Paper 2014-3529, 50th AIAA Joint Propulsion Conference, July 2014.

[Ajmani 2014b] Ajmani, K., and Breisacher, K., "Simulations of NOx Emissions from Low Emissions Discrete Jet Injector Combustor Tests", AIAA Paper 2014-3524, 50th AIAA Joint Propulsion Conference, July 2014.

[Ajmani 2014c] Ajmani, K., Kundu, K., and Yungster, S., "Evaluation of Reduced Mechanisms for Combustion of Jet-A in LDI Combustor CFD Calculations," AIAA Paper 2014-3662, AIAA Propulsion and Energy Conference, Cleveland, OH, July 2014.

[Davoudzadeh 2006] Davoudzadeh, F., Liu, N.-S., and Moder, J.P., "Investigation of Swirling air flows Generated by Axial Swirlers in a Flame Tube," Proceedings of ASME Turbo Expo 2006, GT2006-91300, Barcelona, Spain (also NASA TM 2006-214252)

[Fu 2008] Fu, Y., "Aerodynamics and Combustion of Axial Swirlers," Ph.D. Dissertation, University of Cincinnati, 2008.

[Iannetti 2008] Iannetti, A. C. and Liu, N.-S., "The effect of spray initial conditions on heat release and emissions in LDI CFD calculations"" 46th AIAA Aerospace Science Meeting and Exhibit, Reno, Nevada, AIAA Paper 2008-1150.

[Liu 2011] Liu, N.-S., Shih, T.-H. and Wey, C.T., "Numerical Simulations of Two-Phase Reacting Flow in a Single-Element Lean Direct Injection (LDI) Combustor Using NCC," NASA/TM-2011-217031.

[Liu 2013] Liu, N. -S., Wey, C.T., and Shih, T. -H., "Time-Filtered Navier-Stokes Approach and Emulation of Turbulence-Chemistry Interaction," 51st AIAA Aerospace Sciences Meeting, Dallas TX, AIAA Paper 2013-0707.

[Mongia 2008] Mongia, H. C., 2008, "Recent Progress in Comprehensive Modeling of Gas Turbine Combustion," AIAA Paper 2008-1445.

[Raju 2012] Raju, M. S., “LSPRAY-IV: A Lagrangian Spray Module,” NASA CR-2012-217294.

[Shih 1998] Shih, T.-H., Chen, K.-H., Liu, N.-S., Lumley, J. L., "Modeling of Turbulent Swirling Flows," NASA-TM 1998-113112. 
[Shih 2000] Shih, T.-H., Povinelli, L.A., Liu, N.-S and Chen, K.-H., "Generalized Wall Function for Complex Turbulent Flows," NASA TM 2000-209936.

[Swanson 1997] Swanson, R.C., and Turkel, E., "Multistage Schemes With Multigrid for Euler and NavierStokes Equations," NASA TP-3631, 1997.

[Tacina 2005] Tacina, R., Lee, P., and Wey, C., "A Lean-Direct-Injection Combustor Using a 9 Point Swirl-Venturi Fuel Injector," ISABE 2005-1106.

[Tacina 2014] Tacina, K.M., Chang, C., He, Z.J., Mongia, H.C., Dam, B., and Lee, P., "A Second Generation Swirl-Venturi Lean Direct Injection Combustion Concept," AIAA Paper 2014-3434, AIAA Propulsion and Energy Conference, Cleveland, OH, July 2014.

[Zink 2014] Zink, G.A., Ryon, J.A., and Pack, S.D., "Intermediate Pressure Combustion Research of a Multipoint Low NOx Combustion System," AIAA Paper 2014-3629, AIAA Propulsion and Energy Conference, Cleveland, OH, July 2014. 